the table. These results have been confirmed by repeated tests in which the progress of growth at various concentration-levels of added materials has been followed at frequent intervals for periods as long as 82 hours. Full details of the experiments will appear elsewhere. Attention should be directed to the greater growth of control cultures in stationary tubes than in roller tubes. This has been observed repeatedly. The explanation is not known.

The results strongly indicate that the wound hormone activity of products from injured cells is due to the factor or factors involved in the respiratory mechanism of the cells. The similarity of the spectra of wound hormone preparations to those of adenine nucleotide complexes ${ }^{4}$, the indicated presenc 3 of adenine, pentose and phosphorus, and the absence of pyrimidines ${ }^{5}$ would suggest the possibility that the active principle might be diphospho- or triphosphopyridine nucleotide. Against this possibility there are: (1) negative chemical tests for the pyridine ring ${ }^{4}$ : (2) negative spectral indications of coenzyme I or II on reduction of the preparations with sodium hyposulphite; (3) negative biological tests for coenzyme I in our preparations. The spectral tests of oxidized and reduced preparations were undertaken in collaboration with Prof. Sizer of our department. The biological assay for coenzyme I was carried out through the kindness of Prof. Ball of the Biochemistry Department of Harvard Medical School by Mr. Jandorf of his laboratory. The possibility of the identity of the active factor with other adenyl nucleotide complexes, such as adenine pyrophosphate, yeast adenylic acid and muscle adenylic acid, is being investigated.

JoHN R. LOOFBOURow. AlFred M. WebB. Rosaline K. Abramowitz.

Department of Biology,

Massachusetts Institute of Technology, Cambridge, Massachusetts. Jan. 16.

${ }^{1}$ Loofbourow, Dwyer and Lane, Biochem. $J$., 34, 432 (1940) ; Loofbourow, Dwyer and Cronin, Biochem. J., 35, 603 (1941).

'Loofbourow, Dwyer and Morgan, Studies Inst. Divi Thoma, 1, 137 (1938).

' Fardon, Carroll and Ruddy, Studies Inst. Divi Thoma, 1, 35 (1938) Fardon and Ruddy, Studies Inst. Divi Thomo, 1, 41 (1938) Forris and Ruddy, Studies Inst. Divi Thoma, 1, 53 (1938).

- Sperti, Loofbourow and Dwyer, Studies Inst. Divi Thome, 1, 163 (1937).

- Ioofbourow, Cook and Stimson, NATURE, 142, 573 (1938); Cook Loofbourow and Stimson, Atti $\boldsymbol{X}^{\circ}$ Cong. Intern. Chim., 5, 26 (1939).

\section{A Remarkable Green Line Source}

Is a number of communications in these columns I have reported the observation of high relative intensities of forbidden lines of atomic nitrogen and the forbidden bands of molecular nitrogen in nitrogen afterglows. Both the absolute and relative intensities of these radiations increased with pressure over the range which had been studied up to the spring of 1941. This included pressures from about 0.001 to - $30 \mathrm{~mm}$. In addition, the relative intensities increased as the time between the interruption of the afterglow producing discharge and the exposure of the afterglow was increased ${ }^{1}$. This effect has been referred to as the temporal effect.

One of the tubes that had been used during the previous studies was cleaned and refilled with nitrogen at the high pressure of $100 \mathrm{~mm} .^{2}$. This tube developed a strong afterglow in a remarkedly short time, and in a few days it showed an afterglow so rich in the green auroral line that after the first halfsecond the afterglow was coloured green and the green line could be seen for several seconds in a vision spectroscope. The spectrum which was chosen for reproduction here shows that after the first flash of

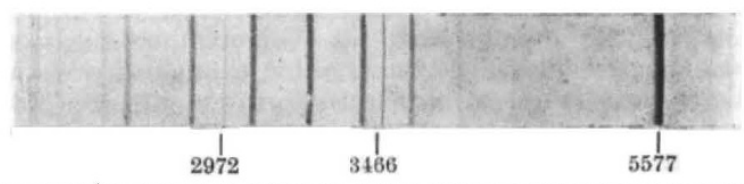

AFTERGLOW SPECTRUM 100 MM. PRESSCRE NITROGEN WITH TRACR OF OXYGEN ; FIRST HALF-SECOND OF AFTERGLOW NOT PHOTOQRAPHED.

the afterglow during which the first-positive and second-positive bands of $\mathrm{N}_{2}$ are considerably weakened, we have an almost monochromatic source of the green auroral line in the visible. The large intensity of Or ' $S_{0}-3 P$, which has about one thirtieth the transition probability of the green line OI ' $S_{0}-{ }^{\prime} D_{2}$, shows in a striking manner the remarkably efficient excitation of the green line. In addition, one should note the large intensity of $\mathrm{Nr} 2 P-4 S$.

Since the discovery of this source, another similar tube at $50 \mathrm{~mm}$. has been prepared, and this, too, shows equally strong forbidden oxygen and nitrogen line excitations. Therefore, the effect must be due to some change in the tube other than pressure. Other features of the afterglow show up as the small amount of oxygen disappears. Among these is the unexpectedly large intensity of the first-negative (auroral) bands of $\mathrm{N}_{2}+$ at both $50 \mathrm{~mm}$. and $100 \mathrm{~mm}$.; the large intensity of the Vegard-Kaplan bands and the large intensity of the green line when much of the oxygen has cleaned up.

These results and a proposed hypothesis to account for these tubes will be presented later, but one conclusion is already tempting, namely, that the catalytic effect of the walls of the tube for the destruction of active nitrogen has in some way been removed and the tube behaves effectively as if it had no valls. The large intensities of the two most striking components of the auroral spectrum, the green line and the auroral bands, lend considerable weight to this conclusion, because both ions and metastable atoms are effectively quenched at walls.

\section{JOSEPH KAPLAN.}

Department of Physics (Meteorology), University of Califormia, Los Angeles, Jan. 5 .

${ }^{1}$ Kaplan, J., Phys. Rev., 57, 662 (1940),

${ }^{2}$ Kaplan, J., Phys. Rev., 54, 176 (1938).

\section{Structure of Vitreous Silica}

Oun attention has recently been directed to a letter from S. S. Lu and Y. L. Chang ${ }^{1}$ concerning the structure of vitreous silica. Two main claims are made. First, that the X-ray diffraction pattern of a thin plate of vitreous silica differs from that of the powdered material and consequently there must be 